\title{
Modeling of Reverberant Radio Channels Using Propagation Graphs
}

\author{
Troels Pedersen, Gerhard Steinböck, and Bernard H. Fleury
}

\begin{abstract}
In measurements of in-room radio channel responses an avalanche effect can be observed: earliest signal components, which appear well separated in delay, are followed by an avalanche of components arriving with increasing rate of occurrence, gradually merging into a diffuse tail with exponentially decaying power. We model the channel as a propagation graph in which vertices represent transmitters, receivers, and scatterers, while edges represent propagation conditions between vertices. The recursive structure of the graph accounts for the exponential power decay and the avalanche effect. We derive a closed form expression for the graph's transfer matrix. This expression is valid for any number of interactions and is straightforward to use in numerical simulations. We discuss an example where time dispersion occurs only due to propagation in between vertices. Numerical experiments reveal that the graph's recursive structure yields both an exponential power decay and an avalanche effect.
\end{abstract}

\section{INTRODUCTION}

Engineering of modern indoor radio systems for communications and geolocation relies heavily on models for the time dispersion of the wideband and ultrawideband radio channels [1]-[3]. From measurement data, as exemplified in Fig. 1 , it appears that the spatial average of the channel impulse response's squared magnitude (referred to as the delay-power spectrum) observed in in-room scenarios exhibits an avalanche effect: The earliest signal components, which appear well separated in time, are followed by an avalanche of components arriving with increasing rate of occurrence, gradually merging into a diffuse tail with exponentially decaying power. The diffuse tail is often referred to as a "dense multipath component" and is commonly attributed to diffuse scattering from rough surfaces and objects which are small compared to the wavelength [5]. A similar avalanche effect is well-known in room acoustics [6] where it is attributed to recursive scattering of sound waves. Indoor radio propagation environments are particularly exposed to recursive scattering as electromagnetic waves may be reflected back and forth in between walls, floor, and ceiling. Thus, the avalanche effect and the exponential power decay may occur due to recursive scattering rather than diffuse scattering.

Recursive scattering phenomena have been previously taken into account in a number of radio channel models. The works [7]-[10] use the analogy to acoustical reverberation theory to predict the exponential decay. As a matter of fact, there exists a well-developed theory of electromagnetic fields in cavities [11], [12], but in this context too the avalanche effect has received little attention. Recursive scattering between particles in a homogeneous medium is a well-known phenomenon

Department of Electronic Systems, Aalborg University, DK-9220 Aalborg East, Denmark. Email: \{troels,gs,fleury\}@es.aau.dk.

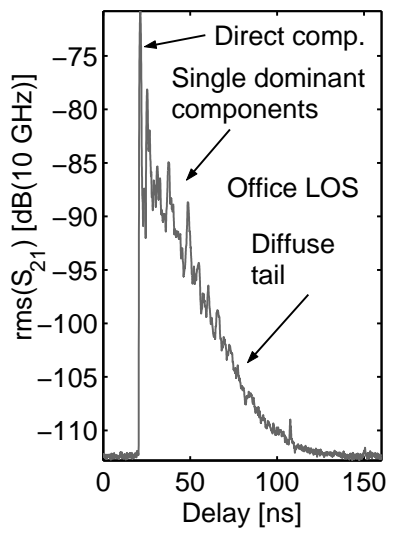

Fig. 1. Spatially averaged delay-power spectrum measured within an office of $5 \times 5 \times 2.6 \mathrm{~m}^{3}$ obtained as the rms value of impulse response for $30 \times 30$ receiver positions on a square horizontal grid with steps of $1 \mathrm{~cm}$. Abscissa includes cable delays; reference to signal bandwidth $(10 \mathrm{GHz})$ shifts ordinate by $-100 \mathrm{~dB}$. Reprinted from [4] with permission (c) 2002 IEEE).

studied by Foldy [13] and Lax [14], [15]. The solution, given by the so-called Foldy-Lax equation [16], has been applied in the context of time-reversal imaging by Shi and Nehorai [17]. The solution is, however, intractable for heterogeneous indoor environments. In [18] the radio propagation mechanism is modeled as a "stream of photons" performing continuous random walks in a homogeneously cluttered environment. The model predicts a delay-power spectrum consisting of a single directly propagating "coherent component" followed by an incoherent tail. Time-dependent radiosity [19]-[22] accounting for delay dispersion has been recently applied to design a model for the received instantaneous power [23]. Thereby, the exponential power decay and the avalanche effect can be predicted.

Simulation studies of communication and localization systems commonly rely on synthetic realizations of the channel impulse response. A multitude of impulse response models exist [2], [3], [24], but only few account for the avalanche effect. The models [25]-[27] treat early components via a geometric model whereas the diffuse tail is generated via another stochastic process; the connection between the propagation environment and the diffuse tail is, however, not considered. Ray tracing methods may also be used to do site-specific simulations [28]. However, ray tracing methods commonly predict the signal energy to be concentrated into the single dominant components whereas the diffuse tail is not well represented.

In this contribution, we model the channel impulse response for the purpose of studying the avalanche effect and, in particular, its relation to recursive scattering. The objective 
is a model which allows for simulation of both realizations of the channel response and average entities such as the delaypower spectrum. Expanding on the previously presented work [29], [30], we propose a unified approach for modeling of the transition from single dominant components to the diffuse tail. The propagation environment is represented in terms of a propagation graph, with vertices representing transmitters, receivers, and scatterers, while edges represent propagation conditions between vertices. The propagation graph accounts inherently for recursive scattering and thus signal components arriving at the receiver may have undergone any number of scatterer interactions. This modelling approach allows for expressing the channel transfer function in closed form for unlimited number of interactions. The formalism enables a virtual "dissection" of the impulse response in so-called partial responses to inspect how recursive scattering leads to a gradual buildup of the diffuse tail.

Propagation graphs may be defined according to a specific scenario for site-specific prediction, or be generated as outcomes of a stochastic process for use in e.g. Monte Carlo simulations. In the present contribution we consider an example of a stochastically generated propagation graph suitable for Monte Carlo simulations. In the example model, scatterer interactions are assumed to cause no time dispersion and thus delay dispersion occurs only due to propagation in between vertices. The simulations reveal that the graph's recursive structure yields both an exponential power decay and an avalanche effect in the generated impulse responses.

\section{REPRESEnTING RADIO ChANNELS AS GRAPHS}

In a typical propagation scenario, the electromagnetic signal emitted by a transmitter propagates through the environment while interacting with a number of objects called scatterers. The receiver, which is usually placed away from the transmitter, senses the electromagnetic signal. If a line of sight exists between the transmitter and the receiver, direct propagation occurs. Also, the signal may arrive at the receiver indirectly via one or more scatterers. In the following we represent propagation mechanisms using graphs allowing for both recursive and non-recursive scattering. We first introduce the necessary definitions of directed graphs.

\section{A. Directed Graphs}

Following [31], we define a directed graph $\mathcal{G}$ as a pair $(\mathcal{V}, \mathcal{E})$ of disjoint sets of vertices and edges. Edge $e \in \mathcal{E}$ with initial vertex denoted by $\operatorname{init}(e)$ and terminal vertex denoted by $\operatorname{term}(e)$ is said to be outgoing from vertex init $(e)$ and ingoing to $\operatorname{term}(e)$. We consider graphs without parallel edges. Thus, there exists no pair of edges $e$ and $e^{\prime}$ such that init $(e)=$ $\operatorname{init}\left(e^{\prime}\right)$ and $\operatorname{term}(e)=\operatorname{term}\left(e^{\prime}\right)$. In this case an edge $e$ can be identified by the vertex pair $(\operatorname{init}(e), \operatorname{term}(e)) \in \mathcal{V}^{2}$ and with a slight abuse of notation we write $e=(\operatorname{init}(e), \operatorname{term}(e))$ and $\mathcal{E} \subseteq \mathcal{V} \times \mathcal{V}$.

\section{B. Propagation Graphs}

We define a propagation graph as a directed graph where vertices represent transmitters, receivers and scatterers. Edges

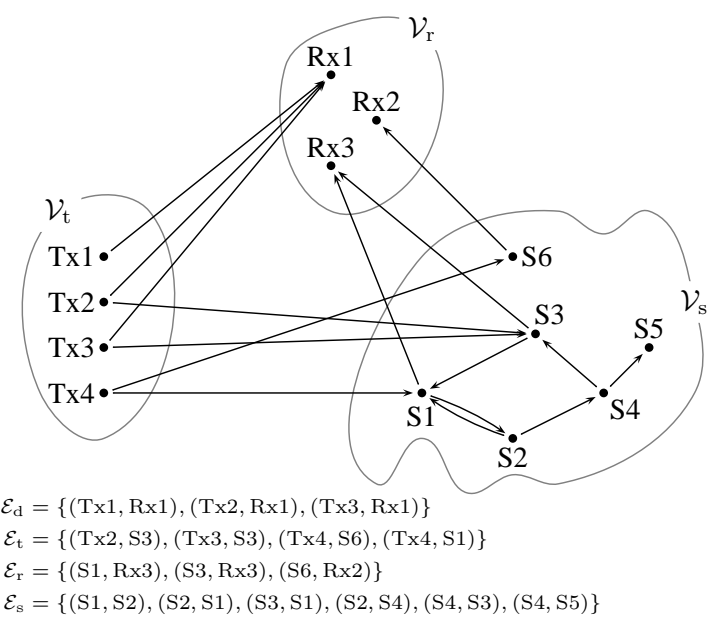

Fig. 2. A propagation graph with four transmit vertices, three receive vertices and six scatterer vertices.

represent the propagation conditions between the vertices. Thus, the vertex set $\mathcal{V}$ of a propagation graph is a union of three disjoint sets: $\mathcal{V}=\mathcal{V}_{\mathrm{t}} \cup \mathcal{V}_{\mathrm{r}} \cup \mathcal{V}_{\mathrm{s}}$, the set of transmitters $\mathcal{V}_{\mathrm{t}}=\left\{\mathrm{Tx} 1, \ldots, \mathrm{Tx} N_{\mathrm{t}}\right\}$, the set of receivers $\mathcal{V}_{\mathrm{r}}=\left\{\mathrm{Rx} 1, \ldots, \mathrm{Rx} N_{\mathrm{r}}\right\}$, and the set of scatterers $\mathcal{V}_{\mathrm{s}}=$ $\left\{\mathrm{S} 1, \ldots, \mathrm{S} N_{\mathrm{S}}\right\}$. The transmit vertices are considered as sources and have only outgoing edges. Likewise, the receivers are considered as sinks with only incoming edges. The edge set $\mathcal{E}$ can thus be partitioned into four disjunct sets as $\mathcal{E}=$ $\mathcal{E}_{\mathrm{d}} \cup \mathcal{E}_{\mathrm{t}} \cup \mathcal{E}_{\mathrm{r}} \cup \mathcal{E}_{\mathrm{s}}$, with direct edges in $\mathcal{E}_{\mathrm{d}}=\mathcal{E} \cap\left(\mathcal{V}_{\mathrm{t}} \times \mathcal{V}_{\mathrm{r}}\right)$, transmitter-scatterer edges in $\mathcal{E}_{\mathrm{t}}=\mathcal{E} \cap\left(\mathcal{V}_{\mathrm{t}} \times \mathcal{V}_{\mathrm{s}}\right)$, scattererreceiver edges in $\mathcal{E}_{\mathrm{r}}=\mathcal{E} \cap\left(\mathcal{V}_{\mathrm{s}} \times \mathcal{V}_{\mathrm{r}}\right)$, and inter-scatterer edges in $\mathcal{E}_{\mathrm{s}}=\mathcal{E} \cap\left(\mathcal{V}_{\mathrm{s}} \times \mathcal{V}_{\mathrm{s}}\right)$. Fig. 2 shows an example propagation graph.

The signals propagate in the graph in the following way. Each transmitter emits a signal that propagates via its outgoing edges. The signal observed by a receiver vertex is the sum of the signals arriving via the ingoing edges. A scatterer sums up the signals on its ingoing edges and re-emits the sum-signal on the outgoing edges. As a signal propagates along an edge, or interacts with a scatterer, it undergoes delay and dispersion in time. The specific delay dispersion endured by a signal depends on the particular propagation mechanism along its edges. Assuming these mechanisms to be linear and time-invariant, this effect can be represented as a convolution with an impulse response or, in the Fourier domain, as a multiplication with a transfer function. Hence, the signal arriving to vertex $v_{n^{\prime}}$ via edge $e=\left(v_{n}, v_{n^{\prime}}\right)$ reads $A_{e}(f) C_{n}(f)$, where $C_{n}(f)$ is the signal emitted by vertex $v_{n}$ and $A_{e}(f)$ denotes the transfer function associated to edge $e$. In other words, the transfer function $A_{e}(f)$ describes the interaction at the initial vertex $v_{n}$ and the propagation from $v_{n}$ to $v_{n^{\prime}}$.

\section{Weighted Adjacency Matrix of a Propagation Graph}

Propagation along the edges is described via a transfer matrix $\mathbf{A}(f)$ which can be viewed as an adjacency matrix with each entry coinciding with the edge transfer function of 


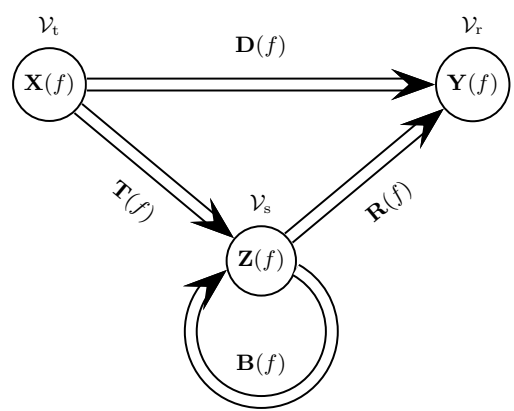

Fig. 3. Vector signal flow graph representation of a propagation graph Vertices represent vertex sets of the propagation graph with associated vector signals. Signal transmission between the sets are represented by the edges and associated transfer matrices.

the corresponding edge. Thus, the weighted adjacency matrix $\mathbf{A}(f) \in \mathbb{C}^{\left(N_{\mathrm{t}}+N_{\mathrm{r}}+N_{\mathrm{s}}\right) \times\left(N_{\mathrm{t}}+N_{\mathrm{r}}+N_{\mathrm{s}}\right)}$ of the propagation graph $\mathcal{G}$ is defined as

$$
[\mathbf{A}(f)]_{n n^{\prime}}= \begin{cases}A_{\left(v_{n}, v_{n^{\prime}}\right)}(f) & \text { if }\left(v_{n}, v_{n^{\prime}}\right) \in \mathcal{E} \\ 0 & \text { otherwise }\end{cases}
$$

i.e., entry $n, n^{\prime}$ of $\mathbf{A}(f)$ is the transfer function from vertex $v_{n}$ to vertex $v_{n^{\prime}}$ of $\mathcal{G}$. Selecting the indexing of the vertices according to

$$
v_{n} \in \begin{cases}\mathcal{V}_{\mathrm{t}}, & n=1, \ldots, N_{\mathrm{t}} \\ \mathcal{V}_{\mathrm{r}}, & n=N_{\mathrm{t}}+1, \ldots, N_{\mathrm{t}}+N_{\mathrm{r}} \\ \mathcal{V}_{\mathrm{s}}, & n=N_{\mathrm{t}}+N_{\mathrm{r}}+1, \ldots, N_{\mathrm{t}}+N_{\mathrm{r}}+N_{\mathrm{s}},\end{cases}
$$

the weighted adjacency matrix takes the form

$$
\mathbf{A}(f)=\left[\begin{array}{ccc}
\mathbf{0} & \mathbf{0} & \mathbf{0} \\
\mathbf{D}(f) & \mathbf{0} & \mathbf{R}(f) \\
\mathbf{T}(f) & \mathbf{0} & \mathbf{B}(f)
\end{array}\right]
$$

where $\mathbf{0}$ denotes the all-zero matrix of the appropriate dimension and the transfer matrices

$$
\begin{array}{ll}
\mathbf{D}(f) \in \mathbb{C}^{N_{\mathrm{r}} \times N_{\mathrm{t}}} & \text { connecting } \mathcal{V}_{\mathrm{t}} \text { to } \mathcal{V}_{\mathrm{r}}, \\
\mathbf{R}(f) \in \mathbb{C}^{N_{\mathrm{r}} \times N_{\mathrm{s}}} & \text { connecting } \mathcal{V}_{\mathrm{s}} \text { to } \mathcal{V}_{\mathrm{r}}, \\
\mathbf{T}(f) \in \mathbb{C}^{N_{\mathrm{s}} \times N_{\mathrm{t}}} & \text { connecting } \mathcal{V}_{\mathrm{t}} \text { to } \mathcal{V}_{\mathrm{s}}, \text { and } \\
\mathbf{B}(f) \in \mathbb{C}^{N_{\mathrm{s}} \times N_{\mathrm{s}}} & \text { interconnecting } \mathcal{V}_{\mathrm{s}} .
\end{array}
$$

The special structure of $\mathbf{A}(f)$ reflects the structure of the propagation graph. The first $N_{\mathrm{t}}$ rows are zero because we do not accept incoming edges into the transmitters. Likewise columns $N_{\mathrm{t}}+1, \ldots, N_{\mathrm{t}}+N_{\mathrm{r}}$ are all zero since the receiver vertices have no outgoing edges.

The input signal vector $\mathbf{X}(f)$ is defined as

$$
\mathbf{X}(f)=\left[X_{1}(f), \ldots, X_{N_{\mathrm{t}}}(f)\right]^{\mathrm{T}},
$$

where $X_{m}(f)$ is the spectrum of the signal emitted by transmitter Txm, and $[\cdot]^{\mathrm{T}}$ denotes the transposition operator. The output signal vector $\mathbf{Y}(f)$ is defined as

$$
\mathbf{Y}(f)=\left[Y_{1}(f), \ldots, Y_{N_{\mathrm{r}}}(f)\right]^{\mathrm{T}}
$$

where $Y_{m}(f)$ is the Fourier transform of the signal observed by receiver $\mathrm{Rx} m$. Similar, to $\mathbf{X}(f)$ and $\mathbf{Y}(f)$ we let $\mathbf{Z}(f)$ denote the output signal vector of the scatterers:

$$
\mathbf{Z}(f)=\left[Z_{1}(f), \ldots, Z_{N_{\mathrm{s}}}(f)\right]^{\mathrm{T}}
$$

with the $n$th entry denoting the Fourier transform of the signal observed at scatterer vertex $\mathrm{S} n$. By the definition of the propagation graph, there are no other signal sources than the vertices in $\mathcal{V}_{\mathrm{t}}$. Assuming linear and time-invariant propagation mechanisms, the input-output relation in the Fourier domain reads

$$
\mathbf{Y}(f)=\mathbf{H}(f) \mathbf{X}(f),
$$

where $\mathbf{H}(f)$ is the $N_{\mathrm{r}} \times N_{\mathrm{t}}$ transfer matrix of the propagation graph.

The structure of the propagation graph unfolds in the vector signal flow graph depicted in Fig. 3. The vertices of the vector signal flow graph represent the three sets $\mathcal{V}_{\mathrm{t}}, \mathcal{V}_{\mathrm{r}}$, and $\mathcal{V}_{\mathrm{s}}$ with the associated signals $\mathbf{X}(f), \mathbf{Y}(f)$, and $\mathbf{Z}(f)$. The edge transfer matrices of the vector signal flow graph are the submatrices of $\mathbf{A}(f)$ defined in (4)-(7).

\section{Transfer MatriX OF A Propagation Graph}

In the following we derive the transfer matrix of a propagation graph. In Subsection III-A we first discuss how the response of a graph is composed of signal contributions propagating via different propagation paths. This representation is, albeit intuitive, impractical for computation of the transfer matrix of graphs with cycles. Thus in Subsections III-B and III-C we give the transfer matrix and partial transfer matrices of a general propagation graph in closed form. Subsection III-D treats the graphical interpretation of reciprocal channels. The section concludes with a discussion of related results in the literature.

\section{A. Propagation Paths and Walks}

The concept of a propagation path is a corner stone in modeling multipath propagation. In the literature, this concept is most often defined in terms of the resulting signal components arriving at the receiver. A shortcoming of this definition is that it is often hard to relate to the propagation environment. The graph terminology offers a convenient alternative. A walk $\ell$ (of length $K$ ) in a graph $\mathcal{G}=(\mathcal{V}, \mathcal{E})$ is defined as a sequence $\ell=\left(v^{(1)}, v^{(2)}, \ldots, v^{(K+1)}\right)$ of vertices in $\mathcal{V}$ such that $\left(v^{(k)}, v^{(k+1)}\right) \in \mathcal{E}, k=1, \ldots K$. We say that $\ell$ is a walk from $v^{(1)}$ to $v^{(K+1)}$ where $v^{(1)}$ is the start vertex and $v^{(K+1)}$ is the end vertex; $v^{(2)}, \ldots, v^{(K)}$ are called the inner vertices of $\ell$. We define a propagation path as a walk in a propagation graph from a transmitter to a receiver. Consequently, all (if any) inner vertices of a propagation path are scatters. A signal that propagates along propagation path $\ell$ traverses $K+1$ (not necessarily different) edges and undergoes $K$ interactions. We refer to such a propagation path as a $K$-bounce path. The zero-bounce path $\ell=\left(v, v^{\prime}\right)$ is called the direct path, from transmitter $v$ to receiver $v^{\prime}$. As an example, referring to the graph depicted in Fig. 2, it is straightforward to verify that $\ell_{1}=(\mathrm{Tx} 1, \mathrm{Rx} 1)$ is a direct path, $\ell_{2}=(\mathrm{Tx} 4, \mathrm{~S} 6, \mathrm{Rx} 2)$ is 
a single-bounce path, and $\ell_{3}=(\mathrm{Tx} 4, \mathrm{~S} 1, \mathrm{~S} 2, \mathrm{~S} 1, \mathrm{Rx} 3)$ is a 3-bounce path

We denote by $\mathcal{L}_{v v^{\prime}}$ the set of propagation paths in $\mathcal{G}$ from transmitter $v$ to receiver $v^{\prime}$. The signal received at $v^{\prime}$ originating from transmitter $v$ is the superposition of signal components each propagating via a propagation path in $\mathcal{L}_{v v^{\prime}}$. Correspondingly, entry $\left(v, v^{\prime}\right)$ of $\mathbf{H}(f)$ reads

$$
H_{v v^{\prime}}(f)=\sum_{\ell \in \mathcal{L}_{v v^{\prime}}} H_{\ell}(f),
$$

where $H_{\ell}(f)$ is the transfer function of propagation path $\ell$.

The number of terms in (12) equals the cardinality of $\mathcal{L}_{v v^{\prime}}$, which may, depending on the structure of the graph, be finite or infinite. As an example, the number of propagation paths is infinite if $v$ and $v^{\prime}$ are connected via a directed cycle in $\mathcal{G}$, i.e. if a propagation path contains a walk from an inner vertex back to itself. The graph in Fig. 2 contains two directed cycles which are connected to both transmitters and receivers.

In the case of an infinite number of propagation paths, computing $H_{v v^{\prime}}(f)$ directly from (12) is infeasible. This problem is commonly circumvented by truncating the sum in (12) to approximate $H_{v v^{\prime}}(f)$ as a finite sum. This approach, however, calls for a method for determining how many terms of the sum should be included in order to achieve reasonable approximation.

In the frequently used " $K$-bounce channel models", propagation paths with more than $K$ interactions are ignored. This approach is motivated by the rationale that at each interaction, the signal is attenuated, and thus terms in (12) resulting from propagation paths with a large number of bounces are weak and can be left out as they do not affect the sum much. This reasoning, however, holds true only if the sum of the components with more than $K$ interactions is insignificant, which may or may not be the case. From this consideration, it is clear that the truncation criterion is non-trivial as it essentially necessitates computation of the whole sum before deciding whether a term can be ignored or not.

\section{B. Transfer Matrix for Recursive and Non-Recursive Propa- gation Graphs}

As an alternative to the approximation methods applied to the sum (12) we now give an exact closed-form expression for the transfer matrix $\mathbf{H}(f)$. Provided that the spectral radius of $\mathbf{B}(f)$ is less than unity, the expression holds true for any number of terms in the sum (12) and thus holds regardless whether the number of propagation paths is finite or infinite.

Theorem 1: If the spectral radius of $\mathbf{B}(f)$ is less than unity, then the transfer matrix of a propagation graph reads

$$
\mathbf{H}(f)=\mathbf{D}(f)+\mathbf{R}(f)[\mathbf{I}-\mathbf{B}(f)]^{-1} \mathbf{T}(f) .
$$

According to Theorem 1 the transfer matrix $\mathbf{H}(f)$ consists of the two following terms: $\mathbf{D}(f)$ representing direct propagation between the transmitters and receivers and $\mathbf{R}(f)[\mathbf{I}-$ $\mathbf{B}(f)]^{-1} \mathbf{T}(f)$ describing indirect propagation. The condition that the spectral radius of $\mathbf{B}(f)$ be less than unity implies that for any vector norm $\|\cdot\|,\|\mathbf{Z}(f)\|>\|\mathbf{B}(f) \mathbf{Z}(f)\|$ for nonzero $\|\mathbf{Z}(f)\|$, cf. [32]. For the Euclidean norm in particular this condition implies the sensible physical requirement that the signal power strictly decreases for each interaction.

Proof: Let $\mathbf{H}_{k}(f)$ denote the transfer matrix for all $k$ bounce propagation paths, then $\mathbf{H}(f)$ can be decomposed as

$$
\mathbf{H}(f)=\sum_{k=0}^{\infty} \mathbf{H}_{k}(f)
$$

where

$$
\mathbf{H}_{k}(f)= \begin{cases}\mathbf{D}(f), & k=0 \\ \mathbf{R}(f) \mathbf{B}^{k-1}(f) \mathbf{T}(f), & k>0 .\end{cases}
$$

Insertion of (15) into (14) yields

$$
\mathbf{H}(f)=\mathbf{D}(f)+\mathbf{R}(f)\left(\sum_{k=1}^{\infty} \mathbf{B}^{k-1}(f)\right) \mathbf{T}(f) .
$$

The infinite sum in (16) is a Neumann series converging to $[\mathbf{I}-\mathbf{B}(f)]^{-1}$ if the spectral radius of $\left.\mathbf{B}(f)\right)$ is less than unity. Inserting this in (16) completes the proof.

The decomposition introduced in (14) makes the effect of the recursive scattering directly visible. The received signal vector is a sum of infinitely many components resulting from any number of interactions. The structure of the propagation mechanism is further exposed by (16) where the emitted vector signal is re-scattered successively in the propagation environment leading to the observed Neumann series. This allows for modeling of channels with infinite impulse responses by expression (13).

It is possible to arrive at (13) in an alternative, but less explicit, manner:

Proof: It is readily observed from the vector signal flow graph in Fig. 3 that $\mathbf{Z}(f)$ can be expressed as

$$
\mathbf{Z}(f)=\mathbf{T}(f) \mathbf{X}(f)+\mathbf{B}(f) \mathbf{Z}(f) .
$$

Since the spectral radius of $\mathbf{B}(f)$ is less than unity we obtain for $\mathbf{Z}(f)$ the solution

$$
\mathbf{Z}(f)=[\mathbf{I}-\mathbf{B}(f)]^{-1} \mathbf{T}(f) \mathbf{X}(f) .
$$

Furthermore, according to Fig. 3 the received signal is of the form

$$
\mathbf{Y}(f)=\mathbf{D}(f) \mathbf{X}(f)+\mathbf{R}(f) \mathbf{Z}(f) .
$$

Insertion of (18) in this expression yields (13).

We remark that the above two proofs allow for propagation paths with any number of bounces. This is highly preferable, as the derived expression (13) is not impaired by approximation errors due to the truncation of the series into a finite number of terms as it occurs when using $K$-bounce models.

A significant virtue of the expression (13) is that propagation effects related to the transmitters and receivers are accounted for in the matrices $\mathbf{D}(f), \mathbf{T}(f)$ and $\mathbf{R}(f)$, but do not affect $\mathbf{B}(f)$. Consequently, the matrix $[\mathbf{I}-\mathbf{B}(f)]^{-1}$ only needs to be computed once even though the configuration of transmitters and receivers changes. This is especially advantageous for simulation studies of e.g. spatial correlation as this leads to a significant reduction in computational complexity. 


\section{Partial Transfer Matrices}

The closed form expression (13) for the transfer matrix of a propagation graph accounts for propagation via an arbitrary number of scatterer interactions. For some applications it is, however, relevant to study only some part of the impulse response associated with a particular number of interactions. One case is where a propagation graph is used to generate only a part of the response and other techniques are used for the remaining parts. Another case is when one must assess the approximation error when the infinite series is truncated. In the following we derive a few useful expressions for such partial transfer matrices.

We define the $K: L$ partial transfer matrix as

$$
\mathbf{H}_{K: L}(f)=\sum_{k=K}^{L} \mathbf{H}_{k}(f), \quad 0 \leq K \leq L,
$$

i.e., we include only contributions from propagation paths with at least $K$, but no more than $L$ bounces. It is straightforward to evaluate (20) for $K=0$, and $L=0,1,2$ :

$$
\begin{aligned}
& \mathbf{H}_{0: 0}(f)=\mathbf{D}(f) \\
& \mathbf{H}_{0: 1}(f)=\mathbf{D}(f)+\mathbf{R}(f) \mathbf{T}(f) \\
& \mathbf{H}_{0: 2}(f)=\mathbf{D}(f)+\mathbf{R}(f) \mathbf{T}(f)+\mathbf{R}(f) \mathbf{B}(f) \mathbf{T}(f) .
\end{aligned}
$$

This expansion of the truncated series is quite intuitive but the obtained expressions are increasingly complex for large $L$. Theorem 2 gives a closed form expression of the partial transfer function $\mathbf{H}_{K, L}(f)$ for arbitrary $K$ and $L$ :

Theorem 2: The partial response $\mathbf{H}_{K: L}(f)$ is given by

$$
\mathbf{H}_{K: L}(f)=\left\{\begin{array}{r}
\mathbf{D}(f)+\mathbf{R}(f)\left[\mathbf{I}-\mathbf{B}^{L}(f)\right][\mathbf{I}-\mathbf{B}(f)]^{-1} \mathbf{T}(f), \\
K=0, L \geq 0 \\
\mathbf{R}(f)\left[\mathbf{B}^{K-1}(f)-\mathbf{B}^{L}(f)\right][\mathbf{I}-\mathbf{B}(f)]^{-1} \mathbf{T}(f), \\
0<K \leq L,
\end{array}\right.
$$

provided that the spectral radius of $\mathbf{B}(f)$ is less than unity.

Proof: The partial transfer function for $0 \leq K \leq L$ reads

$$
\begin{aligned}
\mathbf{H}_{K: L}(f) & =\sum_{k=K}^{\infty} \mathbf{H}_{k}(f)-\sum_{k^{\prime}=L+1}^{\infty} \mathbf{H}_{k^{\prime}}(f) \\
& =\mathbf{H}_{K: \infty}(f)-\mathbf{H}_{L+1: \infty}(f) .
\end{aligned}
$$

For $K=0$ we have $\mathbf{H}_{0: \infty}(f)=\mathbf{H}(f)$ by definition; for $K \geq 1$ we have

$$
\begin{aligned}
\mathbf{H}_{K: \infty}(f) & =\mathbf{R}(f) \sum_{k=K-1}^{\infty} \mathbf{B}^{k}(f) \mathbf{T}(f) \\
& =\mathbf{R}(f) \mathbf{B}^{K-1}(f) \sum_{k=0}^{\infty} \mathbf{B}^{k}(f) \mathbf{T}(f) \\
& =\mathbf{R}(f) \mathbf{B}^{K-1}(f)[\mathbf{I}-\mathbf{B}(f)]^{-1} \mathbf{T}(f) .
\end{aligned}
$$

Inserting (25) into (24) completes the proof.

Theorem 2 enables closed-form computation of $\mathbf{H}_{K: L}(f)$ for any $K \geq L$. We have already listed a few partial transfer matrices in 21), 22), and (23). By definition the partial response $\mathbf{H}_{K: K}(f)$ equals $\mathbf{H}_{K}(f)$ for which an expression is provided in (15). The transfer function of the $K$-bounce approximation is equal to $\mathbf{H}_{0: K}(f)$. Another special case worth mentioning is $\mathbf{H}_{K+1: \infty}(f)=\mathbf{H}(f)-\mathbf{H}_{0: K}(f)$ available from (25), which gives the error due to the $K$-bounce approximation. Thus the validity of the $K$-bounce approximation can be assessed by evaluating some appropriate norm of $\mathbf{H}_{K+1: \infty}(f)$.

\section{Reciprocity and Reverse Graphs}

In most cases, the radio channel is considered reciprocal. As we shall see shortly, the graph terminology accommodates an interesting interpretation of the concept of reciprocity. For any propagation graph we can define the reverse graph in which the roles of transmitter and receiver vertices are swapped. The principle of reciprocity states that the transfer matrix of the reverse channel is equal to the transposed transfer matrix of the forward channel, i.e., a forward channel with transfer matrix $\mathbf{H}(f)$ has a reverse channel with transfer matrix $\tilde{\mathbf{H}}(f)=\mathbf{H}^{\mathrm{T}}(f)$. In the sequel we mark all entities related to the reverse channel with a tilde.

We seek the relation between the forward graph $\mathcal{G}=(\mathcal{V}, \mathcal{E})$ and its reverse $\tilde{\mathcal{G}}=(\tilde{\mathcal{V}}, \tilde{\mathcal{E}})$ under the assumption of reciprocity. More specifically, we are interested in the relation between the weighted adjacency matrix $\mathbf{A}(f)$ of $\mathcal{G}$ and the weighted adjacency matrix $\tilde{\mathbf{A}}(f)$ of $\tilde{\mathcal{G}}$. We shall prove the following theorem:

Theorem 3: For a propagation graph $\mathcal{G}=(\mathcal{V}, \mathcal{E})$ with weighted adjacency matrix $\underset{\tilde{\mathcal{A}}}{\mathbf{A}}(f)$ and transfer matrix $\mathbf{H}(f)$ there exists a reverse graph $\tilde{\mathcal{G}}=(\tilde{\mathcal{V}}, \tilde{\mathcal{E}})$ such that $\tilde{\mathcal{V}}=\mathcal{V}, \tilde{\mathcal{E}}=$ $\left\{\left(v, v^{\prime}\right):\left(v^{\prime}, v\right) \in \mathcal{E}\right\}, \tilde{\mathbf{A}}(f)=\mathbf{A}^{\mathrm{T}}(f)$, and $\tilde{\mathbf{H}}(f)=\mathbf{H}^{\mathrm{T}}(f)$.

Proof: We prove the Theorem by constructing a suitable propagation graph such that the reciprocity condition is fulfilled. We first note that the set of transmitters, receivers, and scatterers is maintained for the reverse channel, thus the vertex set of $\tilde{\mathcal{G}}$ is $\mathcal{V}$ with $\tilde{\mathcal{V}}_{\mathrm{t}}=\mathcal{V}_{\mathrm{r}}, \tilde{\mathcal{V}}_{\mathrm{r}}=\mathcal{V}_{\mathrm{t}}$, and $\tilde{\mathcal{V}}_{\mathrm{s}}=\mathcal{V}_{\mathrm{s}}$. It is immediately clear from the structure of $\mathcal{G}$ that the reverse graph $\tilde{\mathcal{G}}$ has no ingoing edges to $\tilde{\mathcal{V}}_{\mathrm{t}}$ and no outgoing edges from $\tilde{\mathcal{V}}_{\mathrm{r}}$ and $\tilde{\mathcal{G}}$ is thus a propagation graph. Assuming the vertex indexing as in (2), the weighted adjacency matrix of $\tilde{\mathcal{G}}$ is of the form

$$
\tilde{\mathbf{A}}(f)=\left[\begin{array}{ccc}
\mathbf{0} & \tilde{\mathbf{D}}(f) & \tilde{\mathbf{T}}(f) \\
\mathbf{0} & \mathbf{0} & \mathbf{0} \\
\mathbf{0} & \tilde{\mathbf{R}}(f) & \tilde{\mathbf{B}}(f)
\end{array}\right]
$$

where the transfer matrices $\tilde{\mathbf{D}}(f), \tilde{\mathbf{R}}(f), \tilde{\mathbf{T}}(f)$, and $\tilde{\mathbf{B}}(f)$ are defined according to Fig. 4 Equating $\tilde{\mathbf{A}}(f)$ and $\mathbf{A}(f)$ we obtain the identities $\tilde{\mathbf{D}}(f)=\mathbf{D}^{\mathrm{T}}(f), \tilde{\mathbf{B}}(f)=\mathbf{B}^{\mathrm{T}}(f), \tilde{\mathbf{T}}(f)=$ $\mathbf{R}^{\mathrm{T}}(f)$, and $\tilde{\mathbf{R}}(f)=\mathbf{T}^{\mathrm{T}}(f)$. The relation between $\tilde{\mathcal{E}}$ and $\mathcal{E}$ now follows from the definition of the weighted adjacency matrix. The input-output relation of the reverse channel reads $\tilde{\mathbf{Y}}(f)=\tilde{\mathbf{H}}(f) \tilde{\mathbf{X}}(f)$ where $\tilde{\mathbf{X}}(f)$ is the signal vector emitted by the vertices in $\tilde{\mathcal{V}}_{\mathrm{t}}$ and $\tilde{\mathbf{Y}}(f)$ is the signal vector received by the vertices in $\tilde{\mathcal{V}}_{\mathrm{t}}$. Considering Fig. 4 and arguing as in Section III-B yields for the reverse channel

$$
\tilde{\mathbf{H}}(f)=\tilde{\mathbf{D}}(f)+\tilde{\mathbf{R}}(f)[\mathbf{I}-\tilde{\mathbf{B}}(f)]^{-1} \tilde{\mathbf{T}}(f)
$$




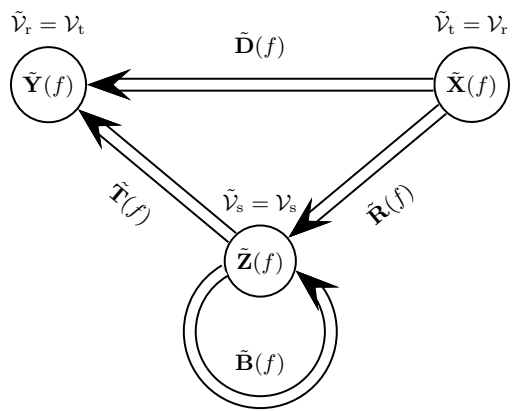

Fig. 4. Vector signal flow graph representation of a reverse propagation graph Compared to the forward graph depicted in Fig. 3 all edges are reversed.

Inserting for $\tilde{\mathbf{D}}(f), \tilde{\mathbf{R}}(f), \tilde{\mathbf{T}}(f)$, and $\tilde{\mathbf{B}}(f)$ leads to

$$
\begin{aligned}
\tilde{\mathbf{H}}(f) & =\mathbf{D}^{\mathrm{T}}(f)+\mathbf{T}^{\mathrm{T}}(f)\left[\mathbf{I}-\mathbf{B}^{\mathrm{T}}(f)\right]^{-1} \mathbf{R}^{\mathrm{T}}(f) \\
& =\mathbf{H}^{\mathrm{T}}(f),
\end{aligned}
$$

where the last equality results from (13).

In words Theorem 3 says that for a propagation graph there exists a reverse graph which fulfills the reciprocity condition. Furthermore, an example of a propagation graph fulfilling the reciprocity condition is the reverse graph $\tilde{\mathcal{G}}$ obtained by reversing all edges of $\mathcal{G}$ while maintaining the edge transfer functions.

\section{E. Related Recursive Scattering Models}

We provide a few examples of recursive models to assist the reader in recognizing models which can be represented by the graphical structure.

In [17] Shi and Nehorai consider a model for recursive scattering between point scatterers in a homogeneous background. The propagation between any point in space is described by a scalar Green's function. The transfer function obtained by applying the Foldy-Lax equation can also be obtained from a propagation graph by defining the sub-matrices of $\mathbf{A}(f)$ as follows. The model does not include a directed term and thus $\mathbf{D}(f)=\mathbf{0}$. The entry of $[\mathbf{T}(f)]_{m_{1} n}$ is the Green's function from transmit vertex $m_{1}$ to scatterer $n$ times the scattering coefficient of scatterer $n^{\prime}$. Similarly, the entry $[\mathbf{R}(f)]_{n m_{2}}$ is the Green's function from the position of scatterer $n$ to receiver $m_{2}$. The entry $[\mathbf{B}(f)]_{n n^{\prime}}, n \neq n^{\prime}$ is the Green's function from the position of scatterer $n$ to the position of scatterer $n^{\prime}$ times the scattering coefficient of scatterer $n$. Since a point scatterer does not scatter back on itself, the diagonal entries of $\mathbf{B}(f)$ are all zero. As can be observed from the above definitions, the assumption of homogeneous background medium leads to the special case with $\mathcal{E}_{\mathrm{d}}=\emptyset, \mathcal{E}_{\mathrm{t}}=\mathcal{V}_{\mathrm{t}} \times \mathcal{V}_{\mathrm{s}}, \mathcal{E}_{\mathrm{r}}=\mathcal{V}_{\mathrm{s}} \times \mathcal{V}_{\mathrm{r}}$, and $\mathcal{E}_{\mathrm{s}}=\mathcal{V}_{\mathrm{s}} \times \mathcal{V}_{\mathrm{s}}$

Another modeling method that can be conveniently described using propagation graphs is (time-dependent) radiosity [19]-[23]. In these methods, the surfaces of the objects in the propagation environment are first divided in smaller patches, then the power transfer between pairs of patches is defined in terms of the so-called "delay-dependent form factor", and finally the power transfer from the transmitter to the receiver is estimated. The delay-dependent form factor is essentially a power impulse response between patches. It appears that for these algorithms no closed-form solution feasible for numerical evaluation is available in the literature. Thus [19][23] resort to iterative solutions which can be achieved after discretizing the inter-patch propagation delays. The timedependent radiosity problem can be expressed in the Fourier domain in terms of a propagation graph where each patch is represented by a scatterer, while the entries of $\mathbf{A}(f)$ are defined according to delay dependent form factor. Using this formulation, a closed form expression of the channel transfer matrix appears immediately by Theorem 1 with no need for quantization of propagation delays.

\section{F. Revisiting Existing Stochastic Radio Channel Models}

It is interesting to revisit existing radio channel models by means of the just defined framework of propagation graphs. Such an effort may reveal some structural differences between models, which are not apparent merely from the mathematical formulation. It is, however, a fact that the interpretation of a transfer function as a propagation graph is not uniqueit is straightforward to construct different propagation graphs which yield the same transfer matrix. Thus different propagation graphs may be associated to the same radio channel model. In the sequel we construct graphs for two well-known stochastic channel models, i.e., the model by Turin et al. [33] and the model by Saleh and Valenzuela [34]. We include here only enough detail to allow for the discussion of the structure of the associated graphs and refer the reader to the original papers for the full description.

The seminal model [33] by Turin et al. can be expressed in the frequency domain as

$$
H(f)=\sum_{\ell=0}^{\infty} \alpha_{\ell} \exp \left(-j 2 \pi f \tau_{\ell}\right),
$$

where $\alpha_{\ell}$ is the complex gain and $\tau_{\ell}$ denotes the delay of component $\ell$. Apart from the direct component, to which we assign index $\ell=0$, the index $\ell$ is merely a dummy index which does not indicate any ordering of terms in (29). The set, $\left\{\left(\tau_{\ell}, \alpha_{\ell}\right): \ell=1,2, \ldots\right\}$ is a marked Poisson point process on $\left[\tau_{0}, \infty\right)$ with complex marks $\left\{\alpha_{\ell}: \ell=1,2, \ldots\right\}$.

The propagation graph we seek should contain a propagation path for each term in the sum (29). We notice that modifying the term $\alpha_{\ell} \exp \left(-j 2 \pi f \tau_{\ell}\right)$ has no effect on the other terms in 29]. This structure can be captured by constructing a propagation graph in which modifying vertices or edges in a propagation path leaves others unchanged. Graph theory offers the convenient notion of "independent walks" [31]: Two or more walks are called independent if none of them contains an inner vertex of another. In particular, propagation paths are independent if they do not traverse the same scatterers. Thus for independent propagation paths $\ell, \ell^{\prime}$, changing path $\ell$ by changing its edge transfer functions or by deleting edges from it, has no effect on path $\ell^{\prime}$. We therefore associate to each term in 29) an independent propagation path. Assuming for simplicity all paths to be single bounce paths this results in the graph shown in Fig. 5 (a). 
a) Turin et al.

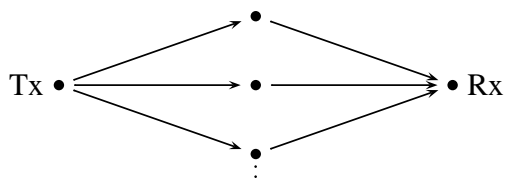

b) Saleh \& Valenzuela

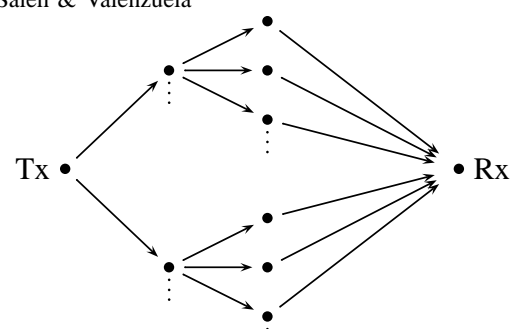

Fig. 5. Propagation graph representations of: a) a realization of the model by Turin et al. [33]; and b) a realization of the Saleh-Valenzuela model [34].

The model by Saleh and Valenzuela [34] can be formulated as a second-order Turin model:

$$
H(f)=\sum_{\ell=0}^{\infty} \exp \left(-j 2 \pi f \tau_{\ell}\right) \sum_{\ell^{\prime}=0}^{\infty} \alpha_{\ell \ell^{\prime}} \exp \left(-j 2 \pi f \tau_{\ell \ell^{\prime}}\right),
$$

where $\ell$ is a cluster index and $\ell^{\prime}$ is a index for the components within a cluster. The set $\left\{\tau_{\ell}: \ell=1,2, \ldots\right\}$ is a Poisson process on $\left[\tau_{0}, \infty\right)$ and conditioned on the cluster delays $\left\{\tau_{\ell} ; \ell=0,1, \ldots\right\}$, the family of sets $\left\{\left(\alpha_{\ell \ell^{\prime}}, \tau_{\ell \ell^{\prime}}\right): \ell^{\prime}=\right.$ $1,2, \ldots\}, \ell=0,1, \ldots$, are independent marked Poisson processes. Using a similar argument as for the Turin model, leads to the graph depicted in Fig. 5(b). The transmitter is connected to a set of super-ordinate scatterers, each scatterer corresponding to a "cluster". These cluster-scatters are then connected to the receiver via independent single-bounce paths passing through sub-ordinate scatterers. In this graph, deleting cluster scatterer $\ell$ makes a whole signal cluster $\ell$ disappear in (30). Similarly, removing the sub-ordinate scatterer $\ell^{\prime}$ the component with index $\ell \ell^{\prime}$ in (30) vanishes.

We end the discussion of the Saleh-Valenzuela model by mentioning that many equivalent graphical interpretations may be given. As an example, one may consider a graph structure as that of the reverse graph of Fig. 5b). In such a structure, the transmitter is directly connected to the sub-ordinate scatterers whereas the receiver is connected to the clusters. Indeed by reversing any number of clusters we obtain an equivalent propagation graph. These graphs share the common property that they contain no cycles.

\section{EXAMPLE: Stochastic Model FOR IN-Room CHANNEL}

The concept of propagation graph introduced until now can be used for describing a broad range of channel models. In this section we apply these general results to a specific example scenario where scatterer interactions are considered to be non-dispersive in delay. This resembles the case where all wave interactions can be considered as specular reflections, or considering points scatterers. The intent is to exemplify that the experimentally observed avalanche effect and diffuse tail can be explained using only scatterer interactions that by themselves yield no delay dispersion. We specify a method feasible for generating such a graph in Monte Carlo simulations. The model discussed in this example is a variant of the model proposed in [29], [30].

\section{A. Weighted Adjacency Matrix}

We define the weighted adjacency matrix according to a geometric model of the environment. We consider a scenario with a single transmitter, a single receiver, and $N_{\mathrm{s}}$ scatterers, i.e., the vertex set reads $\mathcal{V}=\mathcal{V}_{\mathrm{t}} \cup \mathcal{V}_{\mathrm{r}} \cup \mathcal{V}_{\mathrm{s}}$ with $\mathcal{V}_{\mathrm{t}}=\{\mathrm{Tx}\}, \mathcal{V}_{\mathrm{r}}=$ $\{\mathrm{Rx}\}$, and $\mathcal{V}_{\mathrm{S}}=\left\{\mathrm{S} 1, \ldots, \mathrm{S} N_{\mathrm{s}}\right\}$. To each vertex $v \in \mathcal{V}$ we assign a displacement vector $\mathbf{r}_{v} \in \mathbb{R}^{3}$ with respect to a coordinate system with arbitrary origin. To edge $e=\left(v, v^{\prime}\right)$ we associate the Euclidean distance $d_{e}=\left\|\mathbf{r}_{v}-\mathbf{r}_{v^{\prime}}\right\|$, the gain $g_{e}$, the phase $\phi_{e}$, and the propagation delay $\tau_{e}=d_{e} / c$, where $c$ is the speed of light. The edge transfer functions are defined as

$$
A_{e}(f)= \begin{cases}g_{e}(f) \exp j\left(\phi_{e}-2 \pi \tau_{e} f\right) ; & e \in \mathcal{E} \\ 0 ; & e \notin \mathcal{E} .\end{cases}
$$

The edge gains $\left\{g_{e}(f)\right\}$ are defined according to

$$
g_{e}^{2}(f)= \begin{cases}\frac{1}{\left(4 \pi f \tau_{e}\right)^{2}} ; & e \in \mathcal{E}_{\mathrm{d}} \\ \frac{1}{4 \pi f \mu\left(\mathcal{E}_{\mathrm{t}}\right)} \cdot \frac{\tau_{e}^{-2}}{S\left(\mathcal{E}_{\mathrm{t}}\right)} ; & e \in \mathcal{E}_{\mathrm{t}} \\ \frac{1}{4 \pi f \mu\left(\mathcal{E}_{\mathrm{r}}\right)} \cdot \frac{\tau_{e}^{-2}}{S\left(\mathcal{E}_{\mathrm{r}}\right)} ; & e \in \mathcal{E}_{\mathrm{r}} \\ \frac{g^{2}}{\operatorname{odi}(e)^{2}} ; & e \in \mathcal{E}_{\mathrm{s}}\end{cases}
$$

where odi $(e)$ denotes the number of edges from $\operatorname{init}(e)$ to other scatterers and for any $\mathcal{E}^{\prime} \subseteq \mathcal{E}$

$$
\mu\left(\mathcal{E}^{\prime}\right)=\frac{1}{\left|\mathcal{E}^{\prime}\right|} \sum_{e \in \mathcal{E}^{\prime}} \tau_{e} \quad \text { and } \quad S\left(\mathcal{E}^{\prime}\right)=\sum_{e \in \mathcal{E}^{\prime}} \tau_{e}^{-2},
$$

with $|\cdot|$ denoting cardinality. The weight of the direct edge is selected according to the Friis equation [36] assuming isotropic antennas at both ends. The weights of edges in $\mathcal{E}_{\mathrm{t}}$ and $\mathcal{E}_{\mathrm{r}}$ also account for the antenna characteristics. They are computed at the average distance to avoid signal amplification when scatterers are close to a transmitter or receiver, namely when the far-field assumption is invalid. The value of interscatterer gain $g$ is for simplicity assumed fixed.

\section{B. Stochastic Generation of Propagation Graphs}

We now define a stochastic model of the sets $\left\{\mathbf{r}_{v}\right\}, \mathcal{E}$, and $\left\{\phi_{e}\right\}$ as well as a procedure to compute the corresponding transfer function and impulse response. The vertex positions are assumed to reside in a bounded region $\mathcal{R} \subset \mathbb{R}^{3}$ corresponding to the region of interest. The transmitter and receiver positions are assumed to be fixed, while the positions of the $N_{\mathrm{s}}$ scatterers $\left\{\mathbf{r}_{v}: v \in \mathcal{V}_{s}\right\}$ are drawn independently from a uniform distribution on $\mathcal{R}$.

Edges are drawn independently such that a vertex pair $e \in$ $\mathcal{V} \times \mathcal{V}$ is in the edge set $\mathcal{E}$ with probability $P_{e}=\operatorname{Pr}[e \in \mathcal{E}]$ 


1) Draw $\mathbf{r}_{v}, v \in \mathcal{V}_{\mathrm{s}}$ uniformly on $\mathcal{R}$
2) Generate $\mathcal{E}$ according to 34
3) Draw independent phases $\left\{\phi_{e}: e \in \mathcal{E}\right\}$ uniformly on
$[0,2 \pi)$
4) Compute $\mathbf{A}(f)$ within the frequency bandwidth using 31
5) IF spectral radius of $\mathbf{B}(f)$ is larger than unity for some
frequency within the bandwidth THEN GOTO step 1
6) Estimate $H_{K: L}(f)$ and $h_{K: L}(\tau)$ as described in Appendix

Fig. 6. Algorithm for generating full or partial transfer functions and impulse responses for a preselected bandwidth.

defined as

$$
P_{e}= \begin{cases}P_{\mathrm{dir}}, & e=(\mathrm{Tx}, \mathrm{Rx}) \\ 0, & \operatorname{term}(e)=\mathrm{Tx} \\ 0, & \operatorname{init}(e)=\mathrm{Rx} \\ 0, & \operatorname{init}(e)=\operatorname{term}(e) \\ P_{\mathrm{vis}} & \text { otherwise }\end{cases}
$$

The first case of (34) controls the occurrence of a direct component. If $P_{\text {dir }}$ is zero, $D(f)$ is zero with probability one. If $P_{\text {dir }}$ is unity, the direct term $D(f)$ is non-zero with probability one. The second and third cases of (34) exclude ingoing edges to the transmitter and outgoing edges from the receiver. Thus the generated graphs will have the structure defined in Section II-B The fourth case of (34) excludes the occurrence of loops in the graphs. This is sensible as a specular scatterer cannot scatter a signal back to itself. A consequence of this choice is that any realization of the graph is loopless and therefore $\mathbf{A}(f)$ has zeros along its main diagonal. The last case of (34) assigns a constant probability $P_{\mathrm{vis}}$ of the occurrence of edges from $\mathcal{V}_{t}$ to $\mathcal{V}_{s}$, from $\mathcal{V}_{s}$ to $\mathcal{V}_{s}$ and from $\mathcal{V}_{s}$ to $\mathcal{V}_{r}$.

Finally, the phases $\left\{\phi_{e}: e \in \mathcal{E}\right\}$ are drawn independently from a uniform distribution on $[0 ; 2 \pi)$.

Given the deterministic values of parameters $\mathcal{R}, \mathbf{r}_{\mathrm{Tx}}, \mathbf{r}_{\mathrm{Rx}}$, $N_{\mathrm{s}}, P_{\mathrm{dir}}, P_{\mathrm{vis}}$ and $g$, realizations of the (partial) transfer function $H_{K: L}(f)$ and corresponding (partial) impulse response $h_{K: L}(\tau)$ can now be generated for a preselected frequency range $\left[f_{\min }, f_{\max }\right]$, using the algorithm stated in Fig. 6 and Appendix.

\section{Numerical Experiments}

The effect of the recursive scattering phenomenon can now be illustrated by numerical experiments. The parameter settings given in Table \are selected to mimic the experimental setup of [26] used to acquire the measurements reported in Fig. 1 The room size and positions of the transmitter and receiver are chosen as in [26]. We consider the case where direct propagation occurs and set $P_{\text {dir }}$ to unity. The probability of visibility $P_{\mathrm{vis}}$ and the number of scatterers $N_{\mathrm{s}}$ are chosen to mimic the observed avalanche effect. The value of $g$ is set to match the tail slope $\rho \approx-0.4 \mathrm{~dB} / \mathrm{ns}$ of the delay-power spectrum depicted in Fig. 1. The value of $g$ can be related to the slope $\rho$ of the log delay-power spectrum via the approximation $\rho \approx 20 \log _{10}(g) / \mu\left(\mathcal{E}_{\mathrm{s}}\right)$. This
TABLE I

Parameter Settings for the Numerical Examples

\begin{tabular}{lcc}
\hline Parameters & Symbol & Values \\
\hline Room size & $\mathcal{R}$ & {$[0,5] \times[0,5] \times[0,2.6] \mathrm{m}^{3}$} \\
Transmitter position & $\mathbf{r}_{\mathrm{Tx}}$ & {$[1.78,1.0,1.5]^{\mathrm{T}} \mathrm{m}$} \\
Receiver position & $\mathbf{r}_{\mathrm{Rx}}$ & {$[4.18,4.0,1.5]^{\mathrm{T}} \mathrm{m}$} \\
Number of scatterers & $N_{\mathrm{s}}$ & 10 \\
Tail slope & $\rho$ & $-0.4 \mathrm{~dB} / \mathrm{ns}$ \\
Prob. of visibility & $P_{\mathrm{vis}}$ & 0.8 \\
Prob. of direct propagation & $P_{\mathrm{dir}}$ & 1 \\
Speed of light & $c$ & $3.10^{8} \mathrm{~m} / \mathrm{s}$ \\
Transmit signal & $X[m]$ & Unit power Hann pulse \\
Number of frequency samples & $M$ & 8192 \\
\hline
\end{tabular}
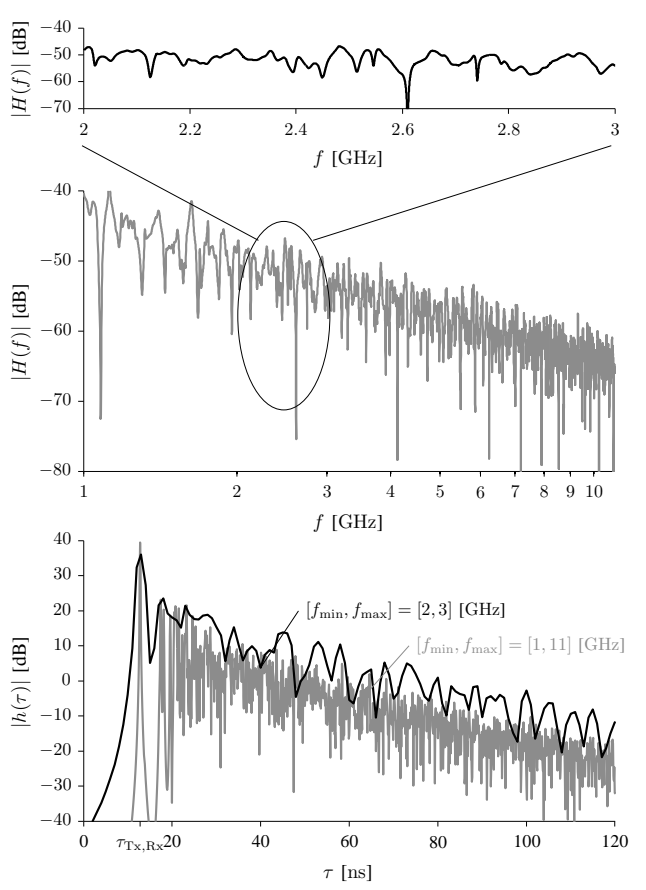

Fig. 7. Channel response for a specific realization of the propagation graph. Top: Transfer function in $\mathrm{dB}\left(20 \log _{10}|H(f)|\right)$. Bottom: Impulse responses in $\mathrm{dB}\left(20 \log _{10}|h(\tau)|\right)$ computed for two frequency bandwidths.

approximation arises by considering the power balance for a scatterer: assuming the signal components arriving at the scatterer to be statistically independent, neglecting the probability of scatterers with outdegree zero, and approximating the delays of edges in $\mathcal{E}_{\mathrm{s}}$ by the average $\mu\left(\mathcal{E}_{\mathrm{s}}\right)$ defined in $33 \mathrm{~b}$.

Fig. 7 shows the amplitude of a single realization of the transfer function. Overall, the squared amplitude of the transfer function decays as $f^{-2}$ due to the definition of $\left\{g_{e}(f)\right\}$. Furthermore, the transfer function exhibits fast fading over the considered frequency band. The lower panel of Fig. 7 reports the corresponding impulse response for two different signal bandwidths. Both impulse responses exhibit an avalanche effect as well as a diffuse tail of which the power decays nearly exponentially with $\rho \approx-0.4 \mathrm{~dB} / \mathrm{ns}$. As anticipated, the transition to the diffuse tail is most visible in the response obtained with the larger bandwidth.

The build up of the impulse response can be examined via the partial impulse responses given in Fig. 8 Inspection of the 

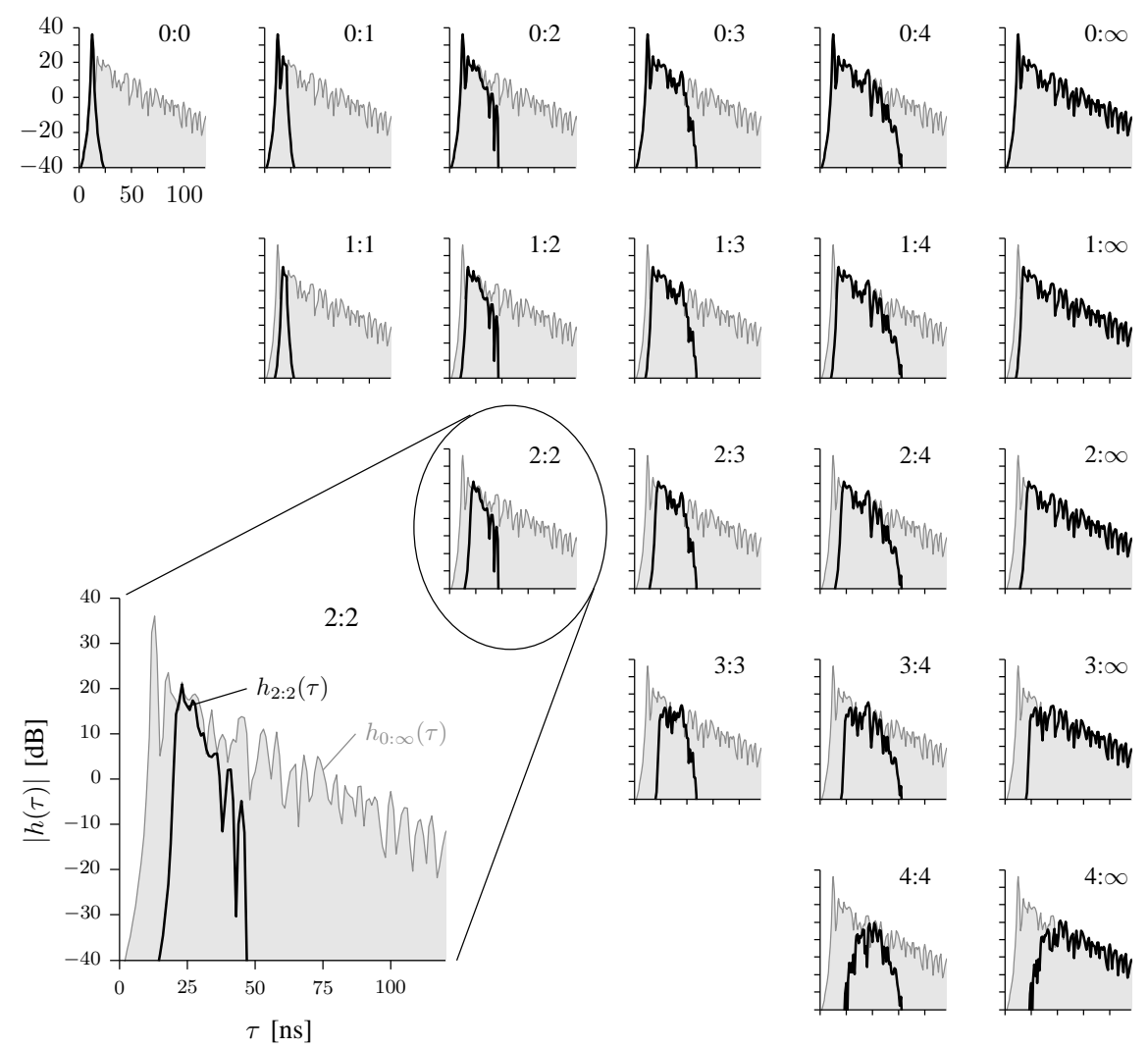

Fig. 8. Partial responses obtained for one graph realization for the bandwidth $[2,3] \mathrm{GHz}$. The $K: L$ settings are indicated in each miniature. Top row: $K$-bounce approximations. Right-most column: error terms resulting from $(K-1)$-bounce approximations. Main diagonal $(K=L)$ : $K$-bounce contributions.

partial responses when $K=L$ reveals that the early part of the tail is due to signal components with a low $K$ while the late part is dominated by higher-order signal components. It can also be noticed that as $K$ increases, the delay at which the maximum of the $K$-bounce partial response occurs and the spread of this response are increasing.

Fig. 9 shows two types of delay-power spectra. The upper panel shows the ensemble average of squared amplitudes of 1000 independently drawn propagation graphs for the two signal bandwidths also considered in Fig. 7) Both spectra exhibit the same trend: A clearly visible peak due to the direct signal is followed by a tail with nearly exponential power decay. As expected, the first peak is wider for the case with $1 \mathrm{GHz}$ bandwidth than for the case with $10 \mathrm{GHz}$ bandwidth. The tails differ by approximately $7 \mathrm{~dB}$. This shift arises due to the $f^{-2}$ trend of the transfer function resulting in a higher received power for the lower frequencies considered in the $1 \mathrm{GHz}$ bandwidth case.

The bottom panel shows spatially averaged delay-power spectra obtained for one particular realization of the propagation graph. The simulated spatial averaged delay-power spectra exhibit the avalanche effect similar to the one observed in Fig. 1. For the $10 \mathrm{GHz}$ bandwidth case the power level of the diffuse tails agrees remarkably well with the measurement in


Fig. 9. Simulated delay-power spectra. Top panel: Ensemble average of 1000 Monte Carlo runs. Bottom panel: Spatial average of a single graph realization assuming the same grid as in Fig. 11 i.e., 900 receiver positions on a $30 \times 30$ horizontal square grid with $1 \times 1 \mathrm{~cm}^{2}$ mesh centered at position $\mathbf{r}_{\mathrm{Rx}}$ given in Table [] 
Fig. 1. The modest deviation of about $3 \mathrm{~dB}$ can be attributed to antenna losses in the measurement.

\section{CONCLUSIONS}

The outset for this work was the observation that the measured impulse responses and delay-power spectra of in-room channels exhibit an avalanche effect: Multipath components appear at increasing rate and gradually merge into a diffuse tail with an exponential decay of power. We considered the question whether the avalanche effect is due to recursive scattering. To this end we modelled the propagation environment as a graph in which vertices represent transmitters, receivers, and scatterers and edges represent propagation conditions between vertices. From this general structure the graph's full and partial frequency transfer matrices were derived in closed form. These expressions can, by specifying the edge transfer functions, be directly used to perform numerical simulations.

We considered as an example a graph-based stochastic model where all interactions are non-dispersive in delay in a scenario similar to an experimentally investigated scenario where the avalanche effect was observed. The impulse responses generated from the model also exhibit an avalanche effect. This numerical experiment lead to the observation that the diffuse tail can be generated even when scatterer interactions are non-dispersive in delay. Furthermore, the exponential decay of the delay-power spectra can be explained by the presence of recursive scattering. As illustrated by the simulation results the proposed model, in contrast to existing models which treat dominant and diffuse components separately, provides a unified account by reproducing the avalanche effect.

\section{APPENDIX}

The transfer function $\mathbf{H}(f)$ and impulse response $\mathbf{h}(\tau)$ can be estimated as follows:

1) Compute $M$ samples of the transfer matrix within the bandwidth $\left[f_{\min }, f_{\max }\right]$

$$
\mathbf{H}[m]=\mathbf{H}\left(f_{\min }+m \Delta_{f}\right), \quad m=0,1, \ldots M-1,
$$

where $\Delta_{f}=\left(f_{\max }-f_{\min }\right) /(M-1)$ and $\mathbf{H}(\cdot)$ is obtained using Theorem 1

2) Estimate the received signal $\mathbf{y}(\tau)$ via the inverse discrete Fourier transform

$$
\begin{array}{r}
\mathbf{y}\left(i \Delta_{\tau}\right)=\Delta_{f} \sum_{m=0}^{M-1} \mathbf{H}[m] \mathbf{X}[m] \exp (j 2 \pi i m / M), \\
i=0, \ldots M-1,
\end{array}
$$

where $\mathbf{X}[m]=\mathbf{X}\left(f_{\min }+m \Delta_{f}\right), m=0,1, \ldots M-1$ and $\Delta_{\tau}=1 /\left(f_{\max }-f_{\min }\right)$.

The impulse response can be estimated by letting $\mathbf{X}[f]$ be a window function of unit power

$$
\left.\int_{f_{\min }}^{f_{\max }}|\mathbf{X}(f)|^{2} \mathrm{~d} f \approx \sum_{m=0}^{M} \mid \mathbf{X}[m]\right]\left.\right|^{2} \Delta_{f}=1,
$$

where $\mathbf{X}[m]=\mathbf{X}\left(f_{\min }+m \Delta_{f}\right)$. The window function must be chosen such that its inverse Fourier transform exhibits a narrow main-lobe and sufficiently low side-lobes; $\mathbf{y}(\tau)$ is then regarded as a good approximation of the impulse response of the channel and by abuse of notation denoted by $\mathbf{h}(\tau)$. Samples of the partial transfer matrix are obtained by replacing $\mathbf{H}(\cdot)$ by $\mathbf{H}_{K: L}(\cdot)$ in (35). The corresponding received partial impulse response is denoted by $\mathbf{h}_{K: L}(\tau)$.

\section{REFERENCES}

[1] H. Hashemi, "Simulation of the urban radio propagation," IEEE Trans. Veh. Technol., vol. 28, pp. 213-225, Aug. 1979.

[2] D. Molkdar, "Review on radio propagation into and within buildings," Microwaves, Antennas and Propagation, IEE Proceedings H, vol. 138, no. 1, pp. 61-73, 1991.

[3] N. Alsindi, B. Alavi, and K. Pahlavan, "Measurement and modeling of ultrawideband TOA-based ranging in indoor multipath environments," IEEE Trans. Veh. Technol., vol. 58, no. 3, pp. 1046-1058, Mar. 2009.

[4] J. Kunisch and J. Pamp, "Measurement results and modeling aspects for the UWB radio channel," in IEEE Conf. on Ultra Wideband Systems and Technologies, 2002. Digest of Papers, May 2002, pp. 19-24.

[5] J. Poutanen, J. Salmi, K. Haneda, V. Kolmonen, and P. Vainikainen, "Angular and shadowing characteristics of dense multipath components in indoor radio channels," IEEE Trans. Antennas Propag., vol. 59, no. 1, pp. 245-253, Jan. 2011.

[6] H. Kuttruff, Room Acoustics. London: Taylor \& Francis, 2000.

[7] C. Holloway, M. Cotton, and P. McKenna, "A model for predicting the power delay profile characteristics inside a room," IEEE Trans. Veh. Technol., vol. 48, no. 4, pp. 1110-1120, July 1999.

[8] R. Rudd and S. Saunders, "Statistical modelling of the indoor radio channel - an acoustic analogy," in Proc. Twelfth International Conf. on Antennas and Propagation (Conf. Publ. No. 491), vol. 1, 31 March-3 April 2003, pp. 220-224.

[9] R. F. Rudd, "The prediction of indoor radio channel impulse response," in The Second European Conf. on Antennas and Propagation, 2007. EuCAP 2007., Nov. 2007, pp. 1-4.

[10] J. B. Andersen, J. Ø. Nielsen, G. F. Pedersen, G. Bauch, and J. M. Herdin, "Room electromagnetics," IEEE Antennas Propag. Mag., vol. 49, no. 2, pp. 27-33, Apr. 2007.

[11] T. H. Lehman, "A statistical theory of electromagnetic fields in complex cavities," Otto von Guericke University of Magdeburg, Tech. Rep., May 1993.

[12] D. A. Hill, Electromagnetic Fields in Cavities: Deterministic and Statistical Theories. Wiley-IEEE Press, 2009.

[13] L. L. Foldy, "The multiple scattering of waves - I. General theory of isotropic scattering by randomly distributed scatterers," Physical Review, vol. 67, pp. 107-119, Feb. 1945.

[14] M. Lax, "Multiple scattering of waves," Reviews of Modern Physics, vol. 23, no. 4, pp. 287-310, Oct. 1951.

[15] - "Multiple scattering of waves. II. The effective field in dense systems," Physical Review, vol. 85, no. 4, pp. 621-629, Feb. 1952.

[16] M. I. Mishchenko, L. D. Travis, and A. A. Lacis, Multiple Scattering of Light by Particles: Radiative Transfer and Coherent Backscattering. Cambridge University Press, May 2006.

[17] G. Shi and A. Nehorai, "Cramér-Rao bound analysis on multiple scattering in multistatic point-scatterer estimation," IEEE Trans. Signal Process., vol. 55, no. 6, pp. 2840-2850, June 2007.

[18] M. Franceschetti, "Stochastic rays pulse propagation," IEEE Trans. Antennas Propag., vol. 52, no. 10, pp. 2742-2752, Oct. 2004.

[19] E.-M. Nosal, M. Hodgson, and I. Ashdown, "Investigation of the validity of radiosity for sound-field prediction in cubic rooms," The Journal of the Acoustical Society of America, vol. 116, no. 6, pp. 3505-3514, 2004. [Online]. Available: http://link.aip.org/link/?JAS/116/3505/1

[20] M. Hodgson and E.-M. Nosal, "Experimental evaluation of radiosity for room sound-field prediction," The Journal of the Acoustical Society of America, vol. 120, no. 2, pp. 808-819, 2006.

[21] S. Siltanen, T. Lokki, S. Kiminki, and L. Savioja, "The room acoustic rendering equation," The Journal of the Acoustical Society of America, vol. 122, no. 3, pp. 1624-1635, 2007.

[22] R. T. Muehleisen and C. W. Beamer, IV, "Steady state acoustic radiosity for the prediction of sound pressure levels in enclosures with diffuse surfaces," Noise Control Engineering Journal, vol. 57, no. 3, pp. 244 262, 2009. 
[23] G. Rougeron, F. G. nd Yannik Gabillet, and K. Boutouch, "Simulation of the indoor propgation of a $60 \mathrm{GHz}$ electromagnetic wave with a time-dependent radiosity algorithm," Computers \& Graphics, vol. 26, pp. 125-141, 2002.

[24] H. Hashemi, "The indoor radio propagation channel," Proc. IEEE, vol. 81, no. 7, pp. 943-968, July 1993.

[25] J. Kunisch and J. Pamp, "UWB radio channel modeling considerations," in Proc. International Conference on Electromagnetics in Advanced Applications 2003, Turin, Sept. 2003.

[26] $\longrightarrow$, "An ultra-wideband space-variant multipath indoor radio channel model," in IEEE Conf. on Ultra Wideband Systems and Technologies, 2003, Nov. 2003, pp. 290-294.

[27] _ - "Locally coherent ultra-wideband radio channel model for sensor networks in industrial environment," in Proc. IEEE 2006 International Conf. on Ultra-Wideband, Sept. 2006, pp. 363-368.

[28] O. Fernandez, L. Valle, M. Domingo, and R. P. Torres, "Flexible rays," IEEE Veh. Technol. Mag., vol. 3, pp. 18-27, Mar. 2008.

[29] T. Pedersen and B. Fleury, "Radio channel modelling using stochastic propagation graphs," in Proc. IEEE International Conf. on Communications ICC '07, June 2007, pp. 2733-2738.

[30] T. Pedersen and B. H. Fleury, "A realistic radio channel model based on stochastic propagation graphs," in Proceedings 5th MATHMOD Vienna 5th Vienna Symposium on Mathematical Modelling, vol. 1,2, Feb. 2006, p. 324, ISBN 3-901608-30-3.

[31] R. Diestel, Graph Theory. Springer-Verlag, 2000.

[32] R. A. Horn and C. A. Johnson, Matrix Analysis. Cambridge University Press, 1985.

[33] G. Turin, F. Clapp, T. Johnston, S. Fine, and D. Lavry, "A statistical model of urban multipath propagation channel," IEEE Trans. Veh. Technol., vol. 21, pp. 1-9, Feb. 1972.

[34] A. A. M. Saleh and R. A. Valenzuela, "A statistical model for indoor multipath propagation channel," IEEE J. Sel. Areas Commun., vol. SAC5, no. 2, pp. 128-137, Feb. 1987.

[35] J. F. C. Kingman, Poisson Processes. Oxford University Press, 1993.

[36] H. T. Friis, "A note on a simple transmission formula," Proceedings of the I.R.E., vol. 34, no. 5, pp. 254-256, may 1946. 